\title{
Cognitive and neural underpinnings of syntactic complexity
}

Diego Fernandez-Duque

Villanova University

Based on a review of the neuroimaging literature, I argue that the resources allocated for processing syntactically complex sentences (i.e., object-extracted relative clauses) are domain-general. Overlapping brain areas are activated by OR clauses and by effortful executive tasks such as storing information in verbal working memory, resolving conflict among competing representations, and switching one's mindset. A re-conceptualization of 'syntactic complexity' in terms of executive functions provides a useful framework in which to explore its links to relational complexity and to cognitive neuroscience. As such, this approach should prove useful to linguists and cognitive scientists alike.

\section{Introduction}

For almost half a century, researchers in psycholinguistics have been interested in the relation between syntactic processing of sentences and domain-general cognitive processes (Miller \& Chomsky, 1963). The question was initially addressed on linguistic and philosophical grounds (Fodor, 1988) and tackled soon thereafter by behavioral and neuropsychological experiments (Caplan \& Waters, 1999; Miyake, Carpenter, \& Just, 1994). Over the last two decades, the emergence of neuroimaging techniques has provided a wealth of information about the relation between language processing and domain-general resources (Grodzinsky \& Friederici, 2006; Kaan \& Swaab, 2002). A comprehensive review of this literature is beyond the scope of the current paper. Instead, I will focus on the processing of relative clauses.

It has been argued that some embedded sentences are more taxing to process than others. In particular, it is often claimed that people have a harder time understanding object-extracted relative $(\mathrm{OR})$ clauses than understanding subject-extracted relative (SR) clauses, as demonstrated by the following example:

OR: The reporter that the senator attacked admitted the mistake SR: The reporter that attacked the senator admitted the mistake

\section{2nd proofs}


OR clauses have a non-canonical word order (object-verb-subject), requiring listeners to reorganize the sequential order of a sentence so that it matches its syntax. In these clauses, the perceptual location of the critical phrase is non-adjacent to its semantic interpretation. Therefore, its processing requires a syntactic movement (ak grammatical transformation) across another element. Some researchers have argued that this additional syntactic operation is at the core of what makes OR clauses more difficult to process. A prominent theory of this kind is the Trace-Deletion Hypothesis (Grodzinsky, 2006). Proponents of this view further argue that syntactic movement has its biological substrate in the left frontal cortex, a claim consistent with some aphasia and neuroimaging data (Ben-Shachar, Hendler, Kahn, Ben-Bashat, \& Grodzinsky, 2003).

In contrast, other researchers have explained the increased processing cost of OR clauses by appealing to 'syntactic complexity.' Syntactic complexity theories differ from each other regarding how complexity is defined. Some are explicit in their definition and provide a metric of complexity that can be use to test predictions (Gibson, 1998; Halford, Wilson, \& Phillips, 1998). Others leave the concept of complexity undefined and focus instead on the experimental conditions that lead to increased complexity (Caplan, Alpert, \& Waters, 1998; Carpenter, Miyake, \& Just, 1994; Friederici, Fiebach, Schlesewsky, Bornkessel, \& von Cramon, 2006). Theories of syntactic complexity also vary from each other on how general are the resources used in syntactic processing. Some theories posit the existence of a limited-capacity memory devoted exclusively to the processing of syntactic relations (Caplan et al., 1998; R. Lewis, 1996). Others argue that syntactic complexity taps onto cognitive resources that are shared with other non-linguistic complex tasks (Andrews, Birney, \& Halford, 2006; Larkin \& Burns, 1977; Miyake et al., 1994).

According to syntactic complexity theories, OR clauses are said to be more difficult to process than SR clauses for many reasons, including the following ones:

1. OR clauses pose a larger storage cost than SR clauses. This is because partially analyzed clauses need to be stored in short-term memory until their completions are available. In other words, the first noun phrase (the reporter) has to be retained in working memory until the verb (attacked) is encountered, at which point syntactic and thematic integration can occur. Once the information is integrated, it becomes part of the text meaning and of the long-term memory representation of that sentence. However, until it is integrated it needs to be held in working memory.

2. OR clauses yield more syntactic ambiguity, as more than one syntactic structure is applicable at the beginning of the sentence. For example, instead of "the reporter the senator attacked admitted the error" one could say "the reporter the senator .and the president disagreed." Comprehension is improved when ambiguity is eliminated by adding the pronoun 'whom', as in "the reporter whom the senator..." (Hakes \& Foss, 1970).

3. OR clauses pose a larger demand for syntactic integration. In OR clauses there is a longer distance between dependents (reporter, attacked) and as additional words are processed, the activation level of the initial element decays. ${ }^{1}$ Therefore more resources are required for the reactivation of the initial element at the time of integration.

4. OR clauses require perspective shifts and therefore pose a larger thematic integration cost (MacWhinney \& Pleh, 1988). In sentences with OR clauses, the firs noun plays two different thematic roles. In our example, 'the reporter' starts as the subject of the main clause, shifts to being the object of the attack in the relative clause, and goes back to being the subject 'who admitted the error' at the end of the sentence. Such perspective shifts during sentence processing mean that the two competing representations have to be coordinated. Comprehension is enhanced when the first noun is an inanimate object, as in "the rock the kid touched was hot" (Hakes, 1972). In such cases, there is no bias toward interpreting the initial noun as a subject; therefore switching to an 'object' representation becomes easier.

It may be useful to compare the processing of syntactic complexity to Executive Function processes, which play a prominent role in theories of working memory, consciousness, and willful action. In most of these theories, one of the properties of executive functioning is its domain-generality. Although the taxonomy of executive function is itself a matter of controversy, executive functions are thought to contribute to:

a. manipulating representations in working memory, as opposed to merely storing them.

b. coordinating ambiguous or conflicting information.

c. switching one's mindset to facilitate the interpretation or implementation of new rules for guiding behavior.

1. According to some theories of syntactic complexity, the distance between dependents is determined not so much by the total number of words to be processed before integration, but rather by the number of new discourse structures (Gibson, 1998). Each time a new discourse referent occurs, cognitive resources have to be deployed to include it in the discourse environment. In contrast, when the new referent is already part of the discourse environment (e.g., indexal pronouns), OR clauses become easy to process (e.g., the book you bought...).

\section{2nd proofs}


From the point of view of a cognitive neuroscientist who specializes in executive function but knows much less about psycholinguistics, the similarities between syntactic complexity of relative clauses and executive function seem, at first sight, quite compelling. On the other hand, these similarities may stem from comparing two very broad and illdefined concepts, rather than from a genuine conceptual overlap. Thus, the remainder of this chapter is devoted to comparing the properties of syntactic complexity of relative clauses and executive function, in an attempt to systematically uncover their possible relation. For this, I will analyze the anatomical overlap between processing syntactic complexity and executive function tasks, with a focus on the frontal cortex. I will also explore the conceptual similarities between tasks that tap syntactic complexity and tasks that tap cognitive complexity in non-syntactic domains.

\section{Neuroimaging of syntactic complexity}

This is not an exhaustive review of the neurology of syntactic complexity but rather a review more limited in scope. From an anatomical standpoint, this review focuses on the inferior gyrus of the frontal cortex, an area that has been implicated in both the processing of syntactic complexity and the processing of many executive function tasks. As a measure of syntactic complexity, the review focuses on the comparison between OR and SR clauses. From a methodological standpoint, the review focuses on neuroimaging research (fMRI, ERP) with only a brief mention of neuropsychological data.

\subsection{Neural activation in OR vs. SR processing, and the influence} of non-syntactic factors

To assess the neural substrates of syntactic complexity, neuroimaging studies have often compared object-extracted and subject-extracted relative clauses. This comparison has consistently shown left hemisphere activation of frontal and temporal areas (Caplan et al., 1998; Just, Carpenter, Keller, Eddy, \& Thulborn, 1996). In the frontal lobe, the activation is centered in the inferior-frontal gyrus (IFG) particularly in its pars opercularis (Brodmann's Area 44) and its pars triangularis (BA 45) Sometimes these areas are referred to as Broca's area, but I will refrain from using this label, as its anatomical boundaries remain elusive and its use is bound to create confusion (Lindenberg, Fangerau, \& Seitz, 2007). ${ }^{2}$ Activation of IFG is consistent with

2. Many studies refer to 'Broca's area' without a clear definition of its anatomical boundaries. Even in studies that do define the boundaries, there is significant variability on what those boundaries are. Part of the reason is that the macroscopic features are not reliable landmarks for its cytoarchitectonic borders (Amunts et al., 1999). To further complicate matters, lesions to 'Broca's area' are neither necessary nor sufficient for the syntactic deficits observed in Broca’s aphasia (Dick et al., 2001) neuropsychological evidence showing that lesion to IFG area reduces comprehension of embedded clauses with non-canonical word order (Friederici, 2002).

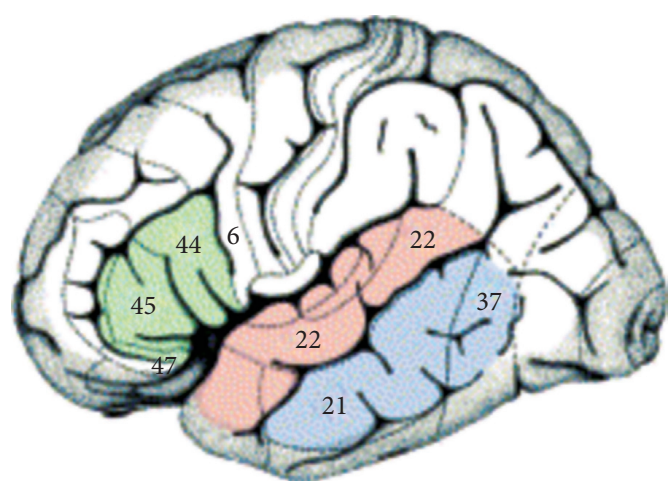

OR clauses differ from SR clauses in terms of syntactic operations, as the canonical word order needs to be reconstructed in the former but not the latter. Thus, one can be tempted to attribute the IFG activation to the extra syntactic processing that OR clauses demand. However, OR and SR clauses also differ in other respects such as the amount of effort involved in information processing. In other words, syntax complexity is confounded with non-syntactic cognitive processes, such as working memory.

To disentangle the contribution of syntax and non-syntactic processes, a useful approach is to include a 'cognitive' factor to the experimental design, and explore whether it interacts with syntactic complexity. The assumption is that computations carried out by overlapping neural substrates will interact. Therefore, the cognitive factor included in the design is usually one known to elicit IFG activation.

Reading low-frequency words causes more activation of left IFG than reading high frequency words. ${ }^{3}$ This raises the question of whether the effect of syntactic complexity in IFG will be modulated by word-frequency. To address this question, OR and SR sentences with high-frequency or low-frequency words were created in a factorial design (Keller, Carpenter, \& Just, 2001). As in previous studies, left IFG showed greater activation for the processing of OR clauses than for the processing

3. Left IFG activation by low frequency-words may be due to lexical selection, as lowfrequency words demand more intense filtering of distracting lures (Badre \& Wagner, 2007; Thompson-Schill, D’Esposito, Aguirre, \& Farah, 1997). Increased IFG activation by low frequency words may also stem from phonological recoding. For example, IFG is activated by reading pseudo-words (i.e., English-like words absent of meaning), a task that requires grapheme-to-phoneme conversion but does not require lexical retrieval. There might even be some amount of anatomical segregation in parts of the IFG between these two mechanisms (Fiebach, Friederici, Muller, \& von Cramon, 2002; Poldrack et al., 1999).

\section{2nd proofs}


of SR clauses, and greater activation for low-frequency words than for sentences with high-frequency words. More importantly, there was an interaction between these two main effects: the effect of syntactic complexity on IFG was evident only for sentences of low-frequency words. These findings point to a common anatomical substrate for the processing of syntactic complexity and the processing of nonsyntactic operations such as phonological recoding and/or lexical selection.

Other fMRI studies have used the factorial design to provide evidence that IFG is not the locus of syntactic movement per se, but rather it supports aspects of working memory. In one such study, syntactic complexity was varied independently from working memory in German indirect wh- questions (Fiebach, Schlesewsky, Lohmann, von Cramon, \& Friederici, 2005). Object-initial questions were compared to subjectinitial questions with the same working memory demands. This comparison did not reveal IFG activation. A different set of results was obtained for sentences with varying working memory load. In some of these sentences the verb was dislocated from its canonical position over a relatively long distance while in others the dislocation was shorter. These two types of sentences had different working memory load. Those with larger working memory load did cause larger IFG activation (BA 44).

In a study of OR vs. SR clause processing, whether the disambiguation occurred early or late within a clause was systematically varied (Fiebach, Vos, \& Friederici, 2004). This early-versus-late manipulation was intended as a manipulation of working memory load. Based on a different measure of working memory capacity, subjects were classified as having high- or low- working memory span. OR clauses caused greater IFG activation than SR clauses, consistent with previous findings. More importantly, the effect of syntactic complexity was dependent on the working memory demands. More specifically, increased IFG activation by syntactically complex sentences was evident only for participants in the low WM span group while reading sentences that demanded most WM (i.e., in which disambiguation occurred late in the clause). In other words, it seems that working memory demands were the main cause of IFG activation.

In sum, there is substantial evidence to argue that IFG is not recruited exclusively for the syntactic reconstruction of canonical word order but rather is implicated in working memory or processing load. As just described, IFG activation by syntactic complexity is modulated by sentence ambiguity, lexical retrieval, and other memory demands.

\subsection{Mechanisms underlying IFG activation in OR clause processing}

In subject-relative clauses, syntax helps to integrate nouns with verbs: once the information is integrated, it becomes part of the long-term memory representation of that sentence. In contrast, in the object-relative clause the partially processed but incomplete syntactic dependencies need to be maintain in working memory. Based on this analysis, working memory differences should begin with the occurrence of the second noun-phrase in the object-relative clause (e.g., 'the reporter that the senator attacked admitted the mistake'). On the other hand, it is at the end of the object-extracted relative clause that syntactic and thematic integrations occur. The verb of the main clause resolves the ambiguity and allows assigning the roles of 'who did what to whom'. If the cost of OR clause processing stems from these syntactic and thematic integrations, the IFG activation should start near the end of the OR clause. These two hypotheses are not necessarily incompatible: it is possible that the IFG activation is driven both by working memory demands and integration costs. Unfortunately, fMRI studies are unable to assess these alternative hypotheses because in fMRI studies the hemodynamic response lags the neuronal response by several seconds, making it impossible to test which part of the sentence is triggering the activation.

This limitation can be overcome using online measures such as gaze duration (Holmes \& O’Regan, 1981; Traxler, Williams, Blozis, \& Morris, 2005), word-byword reading (Gibson, Desmet, Grodner, Watson, \& Ko, 2005), and pupil diameter (Just \& Carpenter, 1993). Studies using these methodologies indicate that the point of greatest effort is at the end of the object-relative clause, when thematic roles are assigned (i.e., when it is decided who did what to whom). Although useful as online measures of performance, these methodologies do not allow direct comparisons to brain activation. This limitation is overcome by event-related scalp potentials (ERPs), as online electrophysiological measures of sentence processing can be correlated with fMRI activation. Although ERPs' spatial resolution is not as good as that of fMRI, its temporal resolution is much superior. Thus, the methodological strengths of the two techniques complement each other very well.

ERP studies provide support for the working memory hypothesis. The electrophysiological response to the OR clause begins to diverge from the SR clause at the appearance of the second noun (the senator) which marks the beginning of a differential working memory load between OR and SR sentences (King \& Kutas, 1995). This divergence occurs in left anterior sites (i.e., frontal lobe) and is similar to the effect found when working memoryload is increased in other types of sentences (Kluender \& Kutas, 1993). Furthermore, the left anterior negativity is also found when comparing SR clauses to unembedded sentences, consistent with the increased memory demands of embedded sentences. Interestingly, the laterality of the OR effect occurs only for reading material; auditory presentation elicits a bilateral effect instead (Muller, King, \& Kutas, 1997). Thus it seems likely that phonological recoding may contribute as a modulator of this effect. Finally, ERP studies also support the integration hypothesis. In fact, the largest ERP difference between OR and SR does occur at the end of the OR clause, when the main clause verb is first displayed (King \& Kutas, 1995).

\section{2nd proofs}




\subsection{Some evidence of syntactic specificity in left IFG activation}

According to the literature reviewed so far, IFG activation while processing OR clauses is mediated by non-linguistic cognitive processes such as working memory and perspective taking. However, some studies argue otherwise. One fMRI study assessed activation under different levels of (a) syntax complexity and (b) speech rate (Peelle, McMillan, Moore, Grossman, \& Wingfield, 2004). It compared OR clauses to SR clauses; speech speed was systematically manipulated. Syntax complexity activated left IFG across all presentation rates. Fast presentation rate elicited medio-frontal activation usually activated by effortful tasks. More importantly, speech rate did not modulate the level of activation due to syntax complexity. This lack of interaction is at odd with the behavioral data in the same task showing that the cost of increased syntax complexity is modulated by speech presentation rate, with larger error rates for OR sentences at faster speech rates. A second study showed activation in left frontal cortex independent of a variety of factors that were manipulated to increase demands (Ben-Shachar et al., 2003). These results have sometimes been interpreted as evidence for a core network of brain regions that supports grammatical processes and includes IFG and postero-lateral temporal cortex (Cooke et al., 2006). Additional brain regions are thought to be engaged as required by extra cognitive demands. It is unclear how best to reconcile these findings and those showing interaction.

\section{Neuroimaging of executive function}

'Executive Function' is an umbrella term for a wide range of functions that contribute to working memory, consciousness, and willful action. A central goal in cognitive science has been to describe how those functions relate to each other and to other cognitive systems (Miyake, Friedman, Emerson, Witzki, \& Howerter, 2000). Cognitive neuroscience has joined the enterprise by exploring whether the same brain areas, most notably in the frontal cortex, are recruited for different aspects of executive control. Although there is not yet a definitive taxonomy of executive function, there is general agreement that a central place should be given to the following abilities:

a. manipulating mental representations in working memory

b. coordinating ambiguous or conflicting information

c. switching mental sets

Some of the experimental paradigms developed over the years aim to explore a single executive function and fractionate it into its more basic subcomponents. Other paradigms aim to relate executive function to other cognitive systems, such as working memory (Baddeley, 1992) and visuospatial attention (Fernandez-Duque \& Posner, 2001).
In such latter cases, 'executive function' is conceptualized as a component of the cognitive system in question. Finally, executive processes are sometimes involved in tasks designed to study some other function. For example, the ability to ignore distractors is an executive function that modulates performance in lexical decision tasks.

Given the central role that verbal working memory seems to play in syntactic complexity, I start by reviewing executive function as part of working memory capacity. This requires a brief description of Working Memory (WM) as a system that allows people to actively maintain and manipulate information. One of most influential models of WM is the one proposed by Baddeley (Baddeley, 1992). That model poses the existence of a system for maintaining verbal information known as the phonological loop, a system for maintaining visual information, and a central executive system for manipulating the information.

\subsection{Simple storage of verbal information in working memory}

For functioning in everyday life, it is absolutely necessary to be able to maintain information after it ceases to be perceptually available. Otherwise, we would be unable to hold a phone number in mind or to understand any sentence more than a few words long. This simple storage of information is dependent on the phonological loop. Presumably, this is the aspect of WM that is tapped by lengthening the distance between syntactic dependencies.

Neuropsychological and neuroimaging evidence shows that the phonological loop is lateralized to the left hemisphere and is further subdivided into a subvocal rehearsal process and a passive storage of phonological information. A recent meta-analysis has confirmed that simple storage of verbal information in working memory activates IFG and that such activation is lateralized to the left hemisphere (Wager \& Smith, 2003). Left IFG is also active during rhyming judgment tasks and other phonological tasks, consistent with a close functional relation between verbal working memory and silent speech (Paulesu, Frith, \& Frackowiak, 1993; Poldrack et al., 1999).

This raises the possibility that increased left IFG activation in response to OR clauses may be due to increased phonological rehearsal. According to this hypothesis, increased complexity would bias participants toward sounding their words out. If this hypothesis is correct, the left IFG activation by OR clauses should disappear under conditions that prevent silent speech (i.e., articulatory suppression). However, some of the evidence suggests otherwise: left IFG activation by OR clauses occurs even when participants read the sentences while uttering an unrelated word every second, aimed at suppressing silent speech (Caplan, Alpert, Waters, \& Olivieri, 2000). Nonetheless, there is some neuropsychological evidence in favor of the hypothesis. For example, comprehension of OR clauses is impaired in patients whose clinical symptoms include effortful speech and dysarthria. One such example is patients with progressive

\section{2nd proofs}


non-fluent aphasia, a type of dementia with brain atrophy most pronounced in left lateral frontal cortex (Grossman \& Moore, 2005).

\subsection{Manipulating information in verbal working memory}

As suggested by the label working memory, people are able not only to store information but also to manipulate and reorganize it. Such ability correlates with individua differences in reasoning, planning, and other intelligent behavior (Kane \& Engle, 2002). The ability to manipulate information in WM has been tested with several different paradigms.

In the $n$-back task, letters are presented one at time separated from each other by a delay of 2 seconds. For each letter, the participant has to decide whether it matches the letter presented $n$ stimuli back. As an example, imagine that in a 2-back task you see the following letter sequence: G, T, L, B, L. Upon seeing the first L, you should report it does not match the reference letter $\mathrm{G}$, that is, the letter that occurred two trials back. As soon as this decision is made, you have to update the information, replacing $\mathrm{G}$ for $\mathrm{T}$ as the reference letter

Although some studies report IFG activation in the 2-back task, it seems likely that such activation is due to increased verbal rehearsal rather than to working memory updating. In support of this interpretation, silent rehearsal tasks cause as much left IFG as 2-back tasks (Awh et al., 1996). This is consistent with the findings from a recent meta-analysis showing that working memory updating does not increase left IFG activation relative to the activation in the simple storage condition (Wager \& Smith, 2003). In the few studies in which updating does activate IFG, the effect is lateralized to the right hemisphere. In sum, it seems that left IFG activation is due to rehearsal rather than updating, at least as far as the n-back task is concerned.

In the alphabetization task, a sequence of letters is presented followed by a delay. During the delay, the participant has to organize the letters in alphabetical order When the probe appears, the subject reports its location in the alphabetical order The alphabetization condition is compared to a storage condition in which the letters have to be retained in the order they are presented. Both conditions equally activate IFG, consistent with a rehearsal interpretation. The alphabetization condition uniquely activates more dorsal areas of the frontal cortex, such as Brodmann's Areas 9 and 46. Thus, working memory manipulation appears to depend on neighboring areas of IFG rather than IFG proper (D’Esposito, Postle, Ballard, \& Lease, 1999).

The Operation Span task assesses the ability to maintain words in memory while solving math problems. In this dual-task paradigm, a sequence of words is presented one at a time, each paired with a math equation. The task requires holding certain information in mind while doing something else. Performance in the Operation Span task correlates with individual differences in reasoning and general fluid intelligence
(Engle, Tuholski, Laughlin, \& Conway, 1999). Activation is more anterior than for syntactic complexity tasks and lateralized to the right hemisphere. It includes Brodmann's Areas 10, 46, and 47 (Wager \& Smith, 2003).

\subsection{Summary of WM activation and its relation to syntactic complexity}

The literature provides support for a common anatomical substrate for syntactic complexity and some aspects of working memory, but not others. On the one hand, there is firm evidence in favor of left IFG activation for subvocal rehearsal and for syntactic complexity. This is consistent with the view that OR clauses require maintaining more information in working memory for longer time. On the other hand, there is less evidence of overlap between syntactic complexity and manipulation of information in working memory. Although syntax complexity and WM manipulation both activate lateral frontal cortex, the precise areas of activation are mostly non-overlapping. However, it remains a possibility that overlap exists with other executive functions. discuss this possibility next.

\subsection{Coordinating ambiguous or conflicting information}

Objects in the environment have many attributes; they have color, shape, motion, size, function, location in space, meaning, etc. For any given task, only a few of those attributes are relevant. Effective information processing requires, among other things, the ability to adequately select which information to process and which to ignore. For example, when picking an apple it is useful to know its size and location, but it is not necessary to know its color. However, color information may become useful when deciding whether to eat the apple, as color will cue the eater to any rotten parts as well as to the flavor she should expect.

The above example illustrates some of the difficulties confronting an agent as she processes a multi-attribute stimulus. On the one hand, some of the perceptually salient attributes carry information that should be ignored because they are useless or even harmful to performance. On the other hand, those same attributes may become relevant at some later point in time. This variability requires that the agent be flexible when deciding how to allocate her attention. In cognitive psychology, the filtering of salient information has been studied under the banner of conflict resolution. The flexible allocation of attention to different dimension has been studied under the banner of set switching. ${ }^{4}$

4. Sometimes both terms are referred to as 'selective attention', a label that highlights that these processes are voluntary, effortful, and require the deployment of domain-general resources.

\section{2nd proofs}




\subsubsection{Conflict resolution in perceptual tasks}

The prime example of conflict resolution is the Stroop task, in which subjects are instructed to respond based on a certain stimulus dimension (e.g., hue) while ignoring some other information (e.g., word meaning). When information from the distracting dimension is incongruent with the target dimension (e.g., the word RED in green ink), conflict arises (Bush, Luu, \& Posner, 2000). Its resolution depends on subjects' ability to ignore the irrelevant information, which in turn requires keeping in mind the correct mindset (i.e., 'respond to hue'). This version of the task is sometimes referred to as the 'verbal' Stroop. Other Stroop-like tasks require the filtering of non-verbal information. The spatial-compatibility task, for example, requires participants to ignore the stimulus location and respond instead based on stimulus shape. The 'flanker task' requires responding to the center target while ignoring distractors on the sides (Fan, Flombaum, McCandliss, Thomas, \& Posner, 2003).

Performance in the verbal Stroop task is impaired following lesion to left IFG (Hamilton \& Martin, 2005) and neuroimaging studies show left lateralized IFG activation for incongruent trials (RED in green ink) (Derrfuss, Brass, Neumann, \& von Cramon, 2005). The left lateralization of these effects is specific to verbal material: conflict in a non-verbal flanker task activates right IFG (Hazeltine, Poldrack, \& Gabrieli, 2000), and lesion to left IFG does not impair performance in the non-verba spatial-compatibility task (Hamilton \& Martin, 2005).

\subsubsection{Conflict resolution in working memory tasks}

In the Stroop and Stroop-like tasks, the filtered information is perceptually available. In contrast, other tasks require the filtering or inhibition of memory representations. Such tasks are relevant to our discussion of syntactic complexity because interpreting a sentence based on new information does require suppressing the no longer relevant interpretation.

One task requiring inhibition of memory representation is the proactive interference task. In this task, a few words (or letters) are presented one per second, immediately followed by a probe. The participant reports whether the probe matches one of the items she just saw. No-match trials can be further divided based on probe familiarity: a probe is said to be familiar if it appeared as an item in the preceding trial. Familiar probes in non-match trials lead to slower and less accurate responses. In those trials, there is conflict between the familiarity of the probe and its absence in the short-term memory set. High conflict trials activate left IFG (Brodmann's area 45); this activation is triggered by the probe onset, which suggests that it is related to conflict resolution (Postle, Brush, \& Nick, 2004). Consistent with this interpretation, performance in high conflict trials is impaired following left IFG lesion (Hamilton \& Martin, 2005). Impairment can also be triggered in healthy adults by temporally inactivating left IFG with the use of repeated transcranial magnetic stimulation (Feredoes, Tononi, \& Postle, 2006).

\subsubsection{Conflict resolution in semantic memory tasks}

When trying to retrieve a word from memory, it is necessary to filter out distractors semantically related to the target. For example, imagine that you are shown a picture of a pencil and asked to name its function. The first word that will come to mind is 'pencil', rather than the correct answer. Or imagine you are shown a picture of an ox and asked to name which animal it is. In this case, a more prototypical member of the category-e.g.,'cow'- may to come to mind. As these examples illustrate, correct performance in these tasks require suppressing the tendency to use the most salient response. Neuropsychological and neuroimaging studies indicate that left IFG plays an important role in such inhibition (Kan \& Thompson-Schill, 2004).

\subsection{Switching mental sets}

When processing multidimensional stimuli, attention is allocated selectively to a particular dimension. However, the focus of attention can be shifted voluntarily to a different stimulus dimension, or even to a different task. For example, a person who had previously been responding based on stimulus color (red, blue) can begin to respond based on stimulus shape (triangle, square). Switching requires selecting the new mental set ('respond to shape') and inhibiting the old one ('respond to color'). It also requires the activation of specific rules ('if red, press left'). These two components of set switching are dissociable in the brain: Set selection activates lateral and medial prefrontal cortex, while rule activation activates mostly the intra-parietal sulcus (Derrfuss et al. 2005; Wager, Jonides, \& Smith, 2006).

A recent meta-analysis found that set switching tasks and verbal Stroop tasks cause overlapping activation in the posterior part of the left IFG (Derrfuss, Brass, \& von Cramon, 2004). In switch trials, this area - known as the inferior frontal junction becomes active even before the appearance of the target (Brass \& von Cramon, 2002). This is consistent with a role in set selection, as behavioral studies have found that rule activation requires a perceptually available target (Monsell, 2003). Furthermore, neuroimaging studies show activation of this brain area at the start of a block of trials, once participants are instructed to get ready for the task (i.e., to adopt the appropriate mindset) (Dosenbach et al., 2006). The set selection is likely to involve the maintenance of task-relevant information, probably in verbal format. Such maintenance of task-relevant information is a process akin to selective attention, the mental highlighting of some stimulus property for the benefit of preferred processing and conscious awareness.

\section{2nd proofs}


One fMRI study provides striking evidence that the left lateralization of set switching is related to verbal processing. In this study, overlapping face/word stimuli were displayed and participants either performed a gender task on the face (male/female) or a syllable-counting task on the word (two syllables or not). Every four trials, a cue signaled participants to continue the same task or switch to the other task. Left IFG was activated when performing the word task and right IFG was activated when performing the face task. More importantly, those effects were larger for switch trials than for repeat trials (Yeung, Nystrom, Aronson, \& Cohen, 2006). In other words, the IFG activation was larger for the trials that required more attention.

Neuropsychological studies have further shown that lesions in the left hemisphere impair performance in switch trials (Mayr, Diedrichsen, Ivry, \& Keele, 2006). Besides causing increased local switch cost (the cost in switch trials), left lateral frontal lesions also lead to increase global switch costs. Global switch costs refer to slow responses to no-switch trials in blocks with bi-dimensional stimuli, relative to blocks with unidimensional stimuli. When the relevance of each dimension alternates every few trials, the irrelevant dimension becomes salient, and more attention is needed to filter it out. If this explanation is correct, we should find that subjects who are least effective at set selec tion are more exposed to the irrelevant dimension and therefore have more conflict to resolve. Neuroimaging studies in normal subjects support this prediction: participants who perform poorly in switch trials show increased activation in some conflict resolution areas of the frontal lobe (superior and middle frontal gyri) (Wager et al., 2006).

Besides being the source of selective attention in some verbal switching tasks, parts of left IFG are also the target of selective attention. For example, when participants have to attend to syntax (e.g., plausibility judgment task), the processing of OR clauses trigger larger IFG activation than when syntax is not task relevant (e.g., detecting the presence of a pseudo-word) (Chen, West, Waters, \& Caplan, 2006).

\section{Coordinating information in relative clauses: A role for conflict} resolution and set switching

In explaining differences between OR and SR clauses, studies of syntactic complexity often appeal to concepts such as 'working memory' or 'cognitive load'. The strength of those explanations lies on a detailed description of what the terms mean and how they relate to syntactic processing. This has been done with some success for some aspects of working memory (Caplan \& Waters, 1999). In contrast, less is known about the conditions under which conflict resolution and set switching would contribute to syntactic processing (Novick, Trueswell, \& Thompson Schill, 2005). Some likely candidates are mentioned next.

Conflict resolution and switching of mindset are likely more involved in the processing of OR clauses than in the processing of SR clauses. One reason for this is that
OR clauses are more ambiguous: in OR clauses, the lexical semantics often do not converge with the default syntactic order. Consider the following two sentences:

a. the girl the boy kicked was big

b. the ball the boy kicked was big.

These are two OR clause sentences, but ' $a$ ' is harder to understand than sentence 'b. The reason for this is that 'girl' is an animate noun (Mak, Vonk, \& Schriefers, 2002). Based on semantic knowledge, the reader builds the expectation that the animate noun will be the agent of the action. Furthermore, in the English language it is very infrequent for an animate noun to be followed by an OR clause (Fox \& Thompson, 1990). Thus, the expectation based on distributional statistics agrees with the expectation based on lexical semantics, namely that following an animate noun the clause will be a SR. In OR clauses, this expectation needs to be overcome, a process that requires conflict resolution.

Consistent with this account, OR sentences in which the first noun is animate elicit greater left IFG activation than OR sentences in which the first noun is inanimate (such as ' $b$ ' above) (Caplan, Chen, \& Waters, in press). It is likely that the activation is elicited at the end of the relative clause. OR sentences require the rapid processing of adjacent verbs, increasing the chances that role assignments for the two clauses will overlap in time. Such temporal overlap is bound to create conflict, as the same noun has to be assigned two different roles (patient of the relative clause, agent of the main clause). This conflict will be greater in the OR sentence with an animate noun in the main clause because in that case there is a stronger expectation that is being violated.

ERPs using a word-by-word reading paradigm reveal that the animacy effect is elicited in part by the relative clause verb, and in part by the main clause verb (Weckerly \& Kutas, 1999). The relative clause verb elicits an effect that is centered in centroparietal sites, with a scalp topography and time course similar to the P600 component. It is probably the neural marker of the mismatch between the incoming stimulus and the current mental set, or possibly a marker of working memory update (Coulson, King, \& Kutas, 1998). ${ }^{5}$ The second ERP component is elicited by the main clause verb.

5. The functional significance of the P600 is a matter of debate. Some researchers argue that it is a general-purpose process related to the updating of information in working memory. In support of this view, the P600 has a scalp distribution similar to that of another ERP component associated with memory updating, the P3b (Coulson et al., 1998). Others argue that the P600 is caused by the syntactic reanalysis that occurs whenever the parser fails to find a meaningful parse (Friederici, 2002). Consistent with this interpretation, the P600 is triggered by syntactic violation and by sentences with correct but non-preferred structure, such as OR sentences and garden-path sentences.

\section{2nd proofs}


It is a left anterior negativity (LAN) that occurs in the 200-500 ms window after stimulus onset. Its location is consistent with IFG activation. Interestingly, the LAN effect is obtained only in participants whose sentence comprehension is good. It would be interesting to explore whether the effect correlates with conflict resolution, set switching, or both.

Support for the claim that conflict resolution is related to syntactic complexity also comes from the observation that OR clauses with indexal pronouns are easy to process. Consider the following two examples:

a. The reporter the senator attacked admitted the error.

b. The reporter you attacked admitted the error.

Sentence (a) is harder to understand than sentence (b). A likely interpretation is that in (a) the embedded subject (the senator) is a new referent in the discourse while in (b) the embedded subject (the pronoun 'you') is already part of the discourse, at least implicitly. According to one of the more prominent theories of syntactic complexity, the presence of a new discourse referent makes more difficult the integration (attacked-reporter) because processing new discourse information demands working memory capacity (Gibson, 1998). As a consequence, access to the main clause noun becomes more difficult and so does the noun-verb integration. The difficulty in accessing the noun is probably due to retrieval interference, although other factors may also come into play (R.L. Lewis, Vasishth, \& Van Dyke, 2006; Van Dyke \& McElree, 2006). To overcome that interference, conflict resolution needs to be applied.

Conflict resolution may also explain why processing OR clauses is more difficult when the noun phrases are similar (Gordon, Hendrick, \& Johnson, 2001). Consider the following two OR clauses:

a. the actor that the director thanked worked in many hit movies before 1980

b. the actor that Fay thanked worked in many hit movies before 1980

Sentence ' $a$ ' is harder to understand than sentence 'b', and participants in a word-byword reading paradigm slow down at the moment of thematic integration (i.e., when processing the verbs of sentence 'a'). In (a) the embedded noun phrase (the director) is similar to the matrix noun phrase (the actor). In (b) the embedded noun phrase is a proper name (Fay) and thus it is less similar and probably causes less interference Once again, it is at the moment of retrieval that such interference is likely to occur (R.L. Lewis et al., 2006; Van Dyke \& McElree, 2006). Consistent with this view, eye tracking studies show that the similarity effect first appears at the moment of processing the verbs (Gordon, Hendrick, Johnson, \& Lee, 2006).

Finally, set switching may help explain the pauses that occur during speech production of relative clauses. According to a prominent view, intonational boundaries are the result of resource processing demands on language production (Watson \& Gibson, 2006). This 'cognitive load' interpretation argues that pauses are due to effortful processing (i.e., executive functions). The claim is that intonational boundaries "provide the speaker with time to (a) plan the properties of upcoming linguistic structure and (b) recover from expending resources after producing complex linguistic structure" (pp. 1045-1046) (Watson, Breen, \& Gibson, 2006). According to this cognitive load hypothesis, intonational boundaries should be most likely to occur before and after long constituents because these locations are likely points for planning and recovery (Watson et al., 2006).

A further prediction would be that intonational boundaries will be most likely for sentences with large set switching demands. Testing this latter prediction might be difficult, as syntactic complexity and set switching are often confounded. Thus, a goal for future research should be to develop experimental sentence comprehension and speech production designs that vary set switching independently from syntactic complexity. Another approach would be to appeal to individual differences, and test whether intonational boundaries are more likely in participants whose set switching skills are below-average. This approach could also be extended to clinical populations that show impairment in these domains. One such group is Parkinson Disease (PD) patients. PD patients are impaired at set switching in both motor and perceptual tasks (Hayes, Davidson, Keele, \& Rafal, 1998). They also have difficulty understanding OR clauses and other syntactically complex sentences. In PD, the deficit in set switching, as well as the deficit in syntactic complexity, is ameliorated by dopamine treatment (Grossman et al., 2001; Hayes et al., 1998). Many patients go on and off medication as part of their treatment, a regimen that offers a rare opportunity to explore the chemical mechanisms underlying syntactic complexity and/or set switching. More generally, PD may prove to be a useful model for testing how executive functions and syntactic complexity interact in the brain. The frontal-striatal loop is a circuit that includes parts of the cerebral cortex as well as sub-cortical structures such as the basal ganglia. This loop is involved in many aspects of behavior, including working memory (Koelsch et al., 2008; McNab \& Klingberg, 2008) and set switching (Crinion et al., 2006), as well as learning (Packard \& Knowlton, 2002), reasoning (Goel, Gold, Kapur, \& Houle, 1997), motor control, and other functions. The hallmark of PD is a dysfunction of this frontal-striatal loop due to partial depletion of the neurotransmitter dopamine. PD deficit in syntactic complexity, such as OR clause comprehension, correlates with deficits in executive tasks such as the Stroop task (Grossman, Lee, Morris,

\section{2nd proofs}


Stern, \& Hurtig, 2002) and set switching tasks (Hochstadt, Nakano, Lieberman, \& Friedman, 2006) ${ }^{6}$

The effects of syntactic complexity and working memory maintenance in PD were explored in an fMRI study using a factorial design (Grossman et al., 2003). Type of relative clause (OR, SR) was used to manipulate syntactic complexity, and length of noun-gap linkage (short-distance dependency, long-distance dependency) was used to manipulate memory storage. In healthy adults, both cortical and sub-cortical components of the loop were recruited by syntactically complex sentences with high memory demand. In $\mathrm{PD}$, those sentences only activated the cortical component (left IFG), failing to activate the subcortical component (striatum). These results are consistent with IFG playing a compensatory role to the subcortical dysfunction. It is unclear whether the compensatory effect is on memory or conflict resolution.

\section{Conceptual similarities between tasks of syntactic} and non-syntactic complexity

The reviewed literature on IFG activity shows commonalities between syntactic complexity and some aspects of working memory and executive function. However, to argue that the syntactic and non-syntactic domains are functionally related it is also important to seek commonalities at other levels of analysis. One promising approach is to compare tasks of syntactic complexity to tasks that share a similar structure, or require a similar set of computations. Tasks of reasoning and intelligence seem likely candidates. I discuss those next.

\subsection{Transitive inference task}

Consider the following scenario:

Premise 1: Mary is taller than Joan.

Premise 2: Joan is taller than Emma.

Conclusion: Therefore, Mary is taller than Emma.

This type of reasoning is relatively easy. If after reading the two premises you were asked who is tallest, you probably would have no difficulty answering "Mary". Now, let's switch the order of the premises:

6. Another factor that may contribute to poor sentence comprehension is PD's slow information processing, as revealed by abnormally late effects of lexical priming in this clinical group.
Premise 2: $\quad$ Mike is taller than Joe.

Premise 1: Ed is taller than Mike.

Conclusion: Therefore, Ed is taller than Joe.

This slight modification makes the second example much more difficult to process. Why? In trying to answer this question, it is worth pointing out that performance in the transitive inference task is correlated with OR clause comprehension (Andrews et al., 2006). Remarkably, the correlation remains significant after controlling for performance in SR clause comprehension, and for performance in a variant of the Operation Span task. This raises the possibility that a common factor underlies performance in OR clause comprehension and transitive inference reasoning.

The factor, it has been proposed, is relational complexity or number of related dimensions that need to be considered simultaneously (Andrews et al., 2006). In the transitive reasoning task, the goal is to rank each person by height, based on relative height information. In the first example, the relations between names can be processed sequentially. This segmentation reduces the task complexity: by the time the second relation is being processed, the first one is already solved. In contrast, in the second example both relations need to be considered simultaneously. In other words, the relational complexity of the task is increased, and so its difficulty.

The processing of SR and OR clauses lends itself to a similar analysis. In this case, the goal is thematic role assignment ('who did what to whom'). Thematic role assignment, which is central to sentence comprehension, requires processing the relation between nouns and verbs. In the SR clause, those relations can be processed sequentially; propositions can be processed one at a time. In contrast, in the OR clause segmentation is more difficult because the verbs, which are needed for assigning the roles, are concentrated at the end of the sentence. Consistent with this interpretation, the largest processing cost in OR sentences occurs at the moment that the verbs are displayed. This is the moment when noun-verb relations are extracted.

Re-describing relative clauses in terms of relational complexity should allow researchers to entrench syntactic complexity into a cognitive literature that includes reasoning and problem solving (Halford \& Andrews, 2004; Halford et al., 1998). For example, the maximum number of relations that can be processed simultaneously is four, according to estimates based experimental research. ${ }^{7}$ There is a developmental

7. The relational complexity of a task can be lowered by segmentation and by conceptua chunking, both of which are important aspects of expertise. Segmentation entails breaking tasks into less complex steps that can be processed serially. Conceptual chunking is the recoding of concepts into less complex relations. For example, 'distance over time' can be recoded as 'speed'. Although useful in reducing complexity, conceptual chunking entails a loss of relational information.

\section{2nd proofs}


progression to this maximum capacity (Andrews \& Halford, 2002). It would be interesting to explore whether the developmental trajectory of relative complexity coincides with the developmental trajectory of relative clause use.

\subsection{Matrix tasks}

When seen through the prism of relational complexity, matrix tasks share a resemblance to the transitive inference task just described. The best known example of this type of task is Raven's Progressive Matrix (RPM), developed in 1938 as a measure of nonverbal intelligence. Over the years, the original version of the task has been adapted to accommodate different populations and methodologies (Carpenter, Just, \& Shell, 1990).

In a version of the task adapted for neuroimaging, a $3 \times 3$ matrix of figures is displayed with the bottom right figure missing (Christoff et al., 2001; Kroger et al., 2002). Participants have to infer the missing figure and select it from among a set of options. The complexity of the problem is based on the number of dimensions that relate figures to each other. For example, a 1-relational problem would vary in only one dimension (e.g., size). In one such problem, the figures might decline in size from left to right. A 2-relational problem would add a second dimension of change (e.g., shade). In this example, besides getting smaller from left to right the figures would also get darker from top to bottom. Solving a 1-relational problem requires evaluating only one dimension, while solving a 2-relational problem requires the simultaneous evaluation of two dimensions. In this sense, the RPM is conceptually similar to the transitive inference task, and to the processing of relative clauses. A comparison of 2-relational vs. 1-relational problems show activation of several brain areas, including anterior part of the left frontal cortex (area 10), posterior part of the IFG (area 44) and sub-cortical parts of the fronto-striatal loop (e.g., basal ganglia) (Christoff et al., 2001; Kroger et al., 2002). ${ }^{8}$

\subsection{Dimensional change card sort task (DCCS)}

This task has been used successfully in the developmental literature to explore the development of executive functions (Zelazo, 2006). Children are asked to sort cards into two piles according to an explicitly stated rule, such as "Play the color game: if it's red, it goes here [to the left], if it is blue it goes here [to the right]". After severa trials the rules change and children are explicitly told to sort based on a dimension

8. The IFG activation is related to the increased difficulty that is associated with more complex trials. In contrast, area 10 of the frontal lobe is specifically activated by relational complexity being active even in 2-relational trials in which the response was quick and accurate. that until then was irrelevant (e.g., 'Now you will play the shape game: if it is a rabbit it goes here [left], if it is a boat it goes here [right]'). At the age of 3, most children are incapable of switching, even though they are able to verbalize the new rule if asked to do so. At the age of 5, most children are capable of performing the task.

These findings have been interpreted in the context of a theory of cognitive complexity and control, which poses that rules are embedded in a hierarchical structure (P.D. Zelazo \& Frye, 1998) . Application of the first-order rules ('if red, left', 'if blue, right'; 'if rabbit, left', 'if boat, right) is contingent on which higher order rule ('sort by color,' 'sort by shape') is active based on the instructions. Although 3-year old children are capable of implementing two first-order rules, it is not until the age of five that they can coordinate them in an embedded structure.

The embedded structure of the DCCS task raises the interesting question of whether it can be performed by people whose language, it has been argued, does not to include embedding (Everett, 2005). Said differently, the DCCS may be a good proxy for assessing the use of embedded structures and rules in speakers of that language. An advantage of the task is the simplicity of its instructions, which can be illustrated without words.

For the purpose of this chapter, this task is relevant in that it requires the coordination of two different yet truthful statements about the very same object. Thus, the task requires flexibility in perspective taking. Something similar may be required for grasping that a noun phrase can be both the subject of the main clause verb and the object of the relative clause verb.

\section{Conclusions}

The starting point of this chapter was the observation that OR clauses are often more difficult to process than SR clauses. In the literature, there are two main interpretations of this finding. One interpretation emphasizes the difference in syntactic operations between SR and OR clauses, arguing that the OR cost and the associated left IFG activation stem from the larger syntactic movement required by OR clauses (Grodzinsky, 2006). The other interpretation emphasizes syntactic complexity, arguing that the OR cost and IFG activation stem from increased demands in cognitive resources such as working memory. Although the bulk of the literature supports this latter interpretation, the exact nature of those resources remains a matter of dispute. Some researchers argue that the resources are for exclusive use in syntactic processing (Caplan \& Waters, 1999) while others claim that the resources are more general, available also to nonsyntactic processing (King \& Kutas, 1995). Finally, some researchers adopt a neutral stance, as if resources were a conceptual primitive in no need of further re-description (Gibson, 1998).

\section{2nd proofs}


The literature reviewed in this chapter challenges the view that the resources for processing OR sentences are syntax-specific: overlapping brain regions are activated by complex tasks in both syntactic and non-syntactic domains. Furthermore, OR comprehension correlates with performance in non-syntactic verbal tasks. These tasks have in common their demands on verbal working memory, conflict resolution, and mental set switching.

Further evidence that the resources for OR comprehension are available beyond syntax comes from neuroimaging studies in which the processing of relative clauses is compared to concatenated sentences (Booth et al., 1999; Just et al., 1996; King \& Kutas, 1995). In those studies, the activation difference between OR and SR clauses has a very similar anatomy to the activation difference between SR and concatenated sentences. This suggests that the activation is not specific to a long syntactic movement across elements; otherwise, the SR clause and the concatenation sentences should have elicited the same amount of activation. The data also argue that the activation is not specific to the processing of embedded sentences; otherwise, OR and SR clauses should have elicited the same amount of activation, as both are embedded.

The most parsimonious interpretation of this common anatomy of SR and OR clauses is, I would argue, that the increased activation in OR processing is driven by the increased difficulty - or the computational processes associated with this increase in difficulty. Of course, this interpretation is in line with the view that the resources are domain-general and closely related to executive functions. In further support of this view, executive function tasks with no syntactic demands often activate areas that overlap those activated by the syntactic task. These effortful tasks may require storing information in verbal working memory, resolving conflict among competitors, or switching one's mindset, as reviewed in earlier sections of this chapter. In al such cases, left IFG is activated, as it is activated in the 'OR vs. SR' and the 'SR vs. concatentation' comparisons.

The re-conceptualization of OR processing in terms of effortful executive functions helps address the underlying difference in the processing of OR and SR clauses. When difficulty between OR and SR clauses is equated, the left IFG activation disappears (Fiebach et al., 2004). This suggests that IFG activation is not triggered by the processing of OR per se, but rather by processes that are often but not always - associated with those clauses.

Arguing that the IFG activation is not due to OR processing per se but rather to the difficulty associated with those clauses may, at first glance, appear to be hair splitting. If OR clauses almost always are more difficult to process than SR clauses then the two explanations for IFG activation would almost always be indistinguish able. This would be a compelling argument if OR clauses were consistently more difficult to process than SR clauses. On closer inspection, there are reasons to doubt this possibility.
For example, when inanimate nouns are used in OR clauses, the OR cost is greatly reduced. This reduction is likely due to the frequent occurrence of those structures in natural language. For example, corpora analyses reveal that when the noun of the main clause is inanimate, the relative clause most likely to follow is an object-extracted clause (Roland, Dick, \& Elman, 2007). Experimental evidence further supports this interpretation. When presented with a sentence that begins with an inanimate noun (e.g., the book that ) and asked to complete it, participants usually choose an objectextracted clause (Gennari \& MacDonald, 2008). ${ }^{9}$ The reverse is true for animate nouns, in which case the sentence is more likely to include a SR clause. This modulatory role of animacy has often been ignored in studies of relative clause processing, which usually include only animate nouns. Ignoring the role of animacy has led to confounding clause type and frequency of use. The latter seems a likely contributor to difficulty of processing, as shown by studies in which executive functions are brought to bear in suppressing the dominant and most frequent interpretation.

More generally, the re-conceptualization of 'syntactic complexity' in terms of executive functions (e.g., conflict resolution, set switching) helps to shed light on the otherwise mysterious concept of 'resources' to which many psycholinguistic studies have appealed when attempting to explain OR processing cost (Fernandez Duque \& Johnson, 2002; Gibson, 1998). It also provides a common framework in which to entrench the study of syntactic complexity with studies of relational complexity and with cognitive neuroscience in general. As such, re-conceptualizing 'syntactic complexity' in terms of executive functions should prove useful to linguists and cognitive scientists alike.

\section{References}

Amunts, K., Schleicher, A., Burgel, U., Mohlberg, H., Uylings, H.B. \& Zilles, K. 1999. Broca’s region revisited: Cytoarchitecture and intersubject variability. The Journal of Comparative Neurology 412(2): 319-341.

Andrews, G., Birney, D. \& Halford, G.S. 2006. Relational processing and working memory capacity in comprehension of relative clause sentences. Memory and Cognition 34(6): 1325-1340.

Andrews, G. \& Halford, G.S. 2002. A cognitive complexity metric applied to cognitive development. Cognitive Psychology 45(2): 153-219.

9. This use of OR clauses is motivated by the speaker's communicative intention to locate the referent of the inanimate noun in conversational space (Ford, Fox, \& Thompson, 2003). Thus, OR clauses often have an "inanimate noun + that + pronoun" structure, with $I$ or you as the pronoun. Of course, the referents of such pronouns have already been established in the discourse and as a consequence, these OR clauses are sometimes processed even more efficiently than SR clauses (Reali \& Christiansen, 2007).

\section{2nd proofs}


Awh, E., Jonides, J., Smith, E.E., Schumacher, E.H., Koeppe, R.A. \& Katz, S. 1996. Dissociation of storage and rehearsal in verbal working memory: Evidence from positron emission tomography. Psychological Science 7(1): 25-31.

Baddeley, A. 1992. Working memory. Science New York, NY 255(5044): 556-559.

Badre, D. \& Wagner, A.D. 2007. Left ventrolateral prefrontal cortex and the cognitive control of memory. Neuropsychologia 45(13): 2883-2901.

Ben-Shachar, M., Hendler, T., Kahn, I., Ben-Bashat, D. \& Grodzinsky, Y. 2003. The neural reality of syntactic transformations: Evidence from functional magnetic resonance imaging. Psychological Science: A Journal of the American Psychological Society/APS 14(5): 433-440.

Booth, J.R., Macwhinney, B., Thulborn, K.R., Sacco, K., Voyvodic, I. \& Feldman, H.M. 1999. Functional organization of activation patterns in children: Whole brain fMRI imaging during three different cognitive tasks. Progress in Neuro-psychopharmacology \& Biological Psychiatry 23(4): 669-682.

Brass, M. \& von Cramon, D.Y. 2002. The role of the frontal cortex in task preparation. Cerebral Cortex New York NY: 1991, 12(9): 908-914.

Bush, G., Luu, P. \& Posner, M.I. 2000. Cognitive and emotional influences in anterior cingulate cortex. Trends in Cognitive Sciences 4(6): 215-222.

Caplan, D., Alpert, N. \& Waters, G. 1998. Effects of syntactic structure and propositional number on patterns of regional cerebral blood flow. Journal of Cognitive Neuroscience 10(4): 541-552.

Caplan, D., Alpert, N., Waters, G. \& Olivieri, A. 2000. Activation of Broca's area by syntactic processing under conditions of concurrent articulation. Human Brain Mapping 9(2): 65-71.

Caplan, D., Chen, E. \& Waters, G. in press. Syntactic and thematic effects on BOLD signal associated with comprehension and determination of plausibility of sentences with relative clauses.

Caplan, D. \& Waters, G.S. 1999. Verbal working memory and sentence comprehension. Behavioral and Brain Sciences 22(1): 77-126.

Carpenter, P.A., Just, M.A. \& Shell, P. 1990. What one intelligence test measures: A theoretical account of the processing in the Raven Progressive Matrices Test. Psychological Review 97(3): 404-431.

Carpenter, P.A., Miyake, A. \& Just, M.A. 1994. Working memory contraints in comprehension: Evidence from individual differences, aphasia, and aging. In Handbook of Psycholin guistics, M.A. Gernsbacher (Ed.), 1075-1122. San Diego CA: Academic Press.

Chen, E., West, W.C., Waters, G. \& Caplan, D. 2006. Determinants of bold signal correlates of processing object-extracted relative clauses. Cortex; A Journal Devoted to the Study of the Nervous System and Behavior 42(4): 591-604.

Christoff, K., Prabhakaran, V., Dorfman, J., Zhao, Z., Kroger, J.K., Holyoak, K.J. et al. 2001. Rostrolateral prefrontal cortex involvement in relational integration during reasoning. NeuroImage 14(5): 1136-1149.

Cooke, A., Grossman, M., DeVita, C., Gonzalez-Atavales, J., Moore, P., Chen, W. et al. 2006. Large-scale neural network for sentence processing. Brain and language 96(1): 14-36.

Coulson, S., King, J. \& Kutas, M. 1998. Expect the unexpected: Event-related brain response to morphosyntactic violations. Language and Cognitive Processes 131: 21-58

Crinion, J., Turner, R., Grogan, A., Hanakawa, T., Noppeney, U., Devlin, J.T. et al. 2006. Language control in the bilingual brain. Science New York NY 312(5779): 1537-1540.

D'Esposito, M., Postle, B.R., Ballard, D. \& Lease, J. 1999. Maintenance versus manipulation of information held in working memory: An event-related fMRI study. Brain and Cognition 41(1): 66-86.
Derrfuss, J., Brass, M., Neumann, J. \& von Cramon, D.Y. 2005. Involvement of the inferior frontal junction in cognitive control: Meta-analyses of switching and Stroop studies. Human Brain Mapping 2(51): 22-34.

Derrfuss, J., Brass, M. \& von Cramon, D.Y. 2004. Cognitive control in the posterior frontolateral cortex: Evidence from common activations in task coordination, interference control, and working memory. NeuroImage 23(2): 604-612.

Dick, F., Bates, E., Wulfeck, B., Utman, J.A., Dronkers, N. \& Gernsbacher, M.A. 2001. Language deficits, localization, and grammar: Evidence for a distributive model of language breakdown in aphasic patients and neurologically intact individuals. Psychological Review 10(84): 759-788.

Dosenbach, N.U., Visscher, K.M., Palmer, E.D., Miezin, F.M., Wenger, K.K., Kang, H.C. et al 2006. A core system for the implementation of task sets. Neuron 50(5): 799-812.

Engle, R.W., Tuholski, S.W., Laughlin, J.E. \& Conway, A.R.A. 1999. Working memory, shortterm memory, and general fluid intelligence: A latent-variable approach. Journal of Experimental Psychology: General 128(3): 309-331.

Everett, D.L. 2005. Cultural constraints on grammar and cognition in Piraha. Current Anthropology 46(4): 621-646.

Fan, J., Flombaum, J.I., McCandliss, B.D., Thomas, K.M. \& Posner, M.I. 2003. Cognitive and brain consequences of conflict. NeuroImage 18(1): 42-57.

Feredoes, E., Tononi, G. \& Postle, B.R. 2006. Direct evidence for a prefrontal contribution to the control of proactive interference in verbal working memory. Proceedings of the National Academy of Sciences of the United States of America 103(51): 19530-19534.

Fernandez-Duque, D. \& Posner, M.I. 2001. Brain imaging of attentional networks in normal and pathological states. Journal of Clinical and Experimental Neuropsychology: Official Journal of the International Neuropsychological Society 23(1): 74-93.

Fernandez Duque, D. \& Johnson, M.L. 2002. Cause and effect theories of attention: The role of conceptual metaphors. Review of General Psychology 6(2): 153-165.

Fiebach, C.J., Friederici, A.D., Muller, K. \& von Cramon, D.Y. 2002. fMRI evidence for dual routes to the mental lexicon in visual word recognition. Journal of Cognitive Neuroscience 14(1): 11-23

Fiebach, C.J., Schlesewsky, M., Lohmann, G. von Cramon, D.Y. \& Friederici, A.D. 2005. Revisiting the role of Broca's area in sentence processing: Syntactic integration versus syntactic working memory. Human brain mapping 24(2): 79-91.

Fiebach, C.J., Vos, S.H. \& Friederici, A.D. 2004. Neural correlates of syntactic ambiguity in sentence comprehension for low and high span readers. Journal of Cognitive Neuroscience 16(9): 1562-1575.

Fodor, J.A. 1983. The Modularity of Mind. Cambridge MA: The MIT Press.

Ford, C.E., Fox, B.A. \& Thompson, S.A. 2003. Social interaction and grammar. In The new psychology of language: Cognitive and Functional Approaches to Language Structure, Vol. 2, M. Tomasello (Ed.), 119-143. Mahwah NJ: Lawrence Erlbaum.

Fox, B.A. \& Thompson, S.A. 1990. A discourse explanation of the grammar or relative clauses in English conversation. Language 66: 297-316.

Friederici, A.D. 2002. Towards a neural basis of auditory sentence processing. Trends in Cognitive Sciences 6(2): 78-84.

Friederici, A.D., Fiebach, C.J., Schlesewsky, M., Bornkessel, I.D. \& von Cramon, D.Y. 2006. Processing linguistic complexity and grammaticality in the left frontal cortex. Cerebral Cortex New York NY 1991 16(12): 1709-1717.

\section{2nd proofs}


Gennari, S.P. \& MacDonald, M.C. 2008. Semantic indeterminacy in object relative clauses. Journal of Memory and Language 58(2): 161-187.

Gibson, E. 1998. Linguistic complexity: Locality of syntactic dependencies. Cognition 68(1): $1-76$.

Gibson, E., Desmet, T., Grodner, D., Watson, D. \& Ko, K. 2005. Reading relative clauses in English. Cognitive Linguistics 16(2): 313-353.

Goel, V., Gold, B., Kapur, S. \& Houle, S. 1997. The seats of reason? An imaging study of deductive and inductive reasoning. Neuroreport, 8(5): 1305-1310

Gordon, P.C., Hendrick, R. \& Johnson, M. 2001. Memory interference during language processing Journal of Experimental Psychology: Learning, Memory, and Cognition 27(6): 1411-1423.

Gordon, P.C., Hendrick, R., Johnson, M. \& Lee, Y. 2006. Similarity-based interference during language comprehension: Evidence from eye tracking during reading. Journal of Experimental Psychology: Learning, Memory, and Cognition, 32(6): 1304-1321.

Grodzinsky, Y. 2006. A blueprint for a brain map of syntax. In Broca's Region, Y. Grodzinsky \& K. Amunts (Eds), 83-107. Oxford: OUP.

Grodzinsky, Y. \& Friederici, A.D. 2006. Neuroimaging of syntax and syntactic processing. Current Opinion in Neurobiology 16(2): 240-246.

Grossman, M., Cooke, A., DeVita, C., Lee, C., Alsop, D., Detre, J. et al. 2003. Grammatica and resource components of sentence processing in Parkinson's disease: An fMRI study. Neurology 60(5): 775-781.

Grossman, M., Glosser, G., Kalmanson, I., Morris, J., Stern, M.B. \& Hurtig, H.I. 2001. Dopamine supports sentence comprehension in Parkinson's Disease. Journal of the Neurological Sciences, 184(2): 123-130.

Grossman, M., Lee, C., Morris, J., Stern, M.B. \& Hurtig, H.I. 2002. Assessing resource demands during sentence processing in Parkinson's disease. Brain and language 80(3): 603-616.

Grossman, M. \& Moore, P. 2005. A longitudinal study of sentence comprehension difficulty in primary progressive aphasia. Journal of Neurology, Neurosurgery, and Psychiatry 76(5): 644-649.

Hakes, D.T. 1972. Effects of reducing complement constructions on sentence comprehension. Journal of Verbal Learning and Verbal Behavior 11(3): 278-286.

Hakes, D.T. \& Foss, D.J. 1970. Decision processes during sentence comprehension: Effects of surface structure reconsidered. Perception and Psychophysics 8(6): 413-416.

Halford, G.S. \& Andrews, G. 2004. The development of deductive reasoning: How important is complexity? Thinking and Reasoning 10(2): 123-145.

Halford, G.S., Wilson, W.H. \& Phillips, S. 1998. Processing capacity defined by relational complexity: Implications for comparative, developmental, and cognitive psychology. The Behavioral and Brain Sciences 21(6): 803-831; discussion 831-864.

Hamilton, A.C. \& Martin, R.C. 2005. Dissociations among tasks involving inhibition: A singlecase study. Cognitive, Affective \& Behavioral Neuroscience 5(1): 1-13.

Hayes, A.E., Davidson, M.C., Keele, S.W. \& Rafal, R.D. 1998. Toward a functional analysis of the basal ganglia. Journal of Cognitive Neuroscience 10(2): 178-198.

Hazeltine, E., Poldrack, R. \& Gabrieli, J.D. 2000. Neural activation during response competition. Journal of Cognitive Neuroscience 12 (Suppl 2): 118-129.

Hochstadt, J., Nakano, H., Lieberman, P. \& Friedman, J. 2006. The roles of sequencing and verbal working memory in sentence comprehension deficits in Parkinson's disease. Brain and language 97(3): 243-257.

Holmes, V.M. \& O’Regan, J.K. 1981. Eye fixation patterns during the reading of relative-clause sentences. Journal of Verbal Learning and Verbal Behavior 20(4): 417-430.
Just, M.A. \& Carpenter, P.A. 1993. The intensity dimension of thought: Pupillometric indices of sentence processing. Canadian Journal of Experimental Psychology 47(2): 310-339.

Just, M.A., Carpenter, P.A., Keller, T.A., Eddy, W.F. \& Thulborn, K.R. 1996. Brain activation modulated by sentence comprehension. Science New York NY 274(5284): 114-116.

Kaan, E. \& Swaab, T.Y. 2002. The brain circuitry of syntactic comprehension. Trends in Cognitive Sciences 6(8): 350-356.

Kan, I.P. \& Thompson-Schill, S.L. 2004. Effect of name agreement on prefrontal activity during overt and covert picture naming. Cognitive, Affective \& Behavioral Neuroscience 4(1): 43-57.

Kane, M.J. \& Engle, R.W. 2002. The role of prefrontal cortex in working-memory capacity, executive attention, and general fluid intelligence: An individual-differences perspective. Psychonomic Bulletin \& Review 9(4): 637-671.

Keller, T.A., Carpenter, P.A. \& Just, M.A. 2001. The neural bases of sentence comprehension: A fMRI examination of syntactic and lexical processing. Cerebral Cortex New York NY: 1991, 11(3): 223-237.

King, J.W. \& Kutas, M. 1995. Who did what and when? Using word- and clause-level ERPs to monitor working memory usage in reading. Journal of Cognitive Neuroscience 7(3): 376-395.

Kluender, R. \& Kutas, M. 1993. Bridging the gap: Evidence from ERPs on the processing of unbounded dependencies. Journal of Cognitive Neuroscience 5(2): 196-214.

Koelsch, S., Schulze, K., Sammler, D., Fritz, T., Muller, K. \& Gruber, O. 2008. Functional architecture of verbal and tonal working memory: An FMRI study. Human Brain Mapping.

Kroger, J.K., Sabb, F.W., Fales, C.L., Bookheimer, S.Y., Cohen, M.S. \& Holyoak, K.J. 2002. Recruitment of anterior dorsolateral prefrontal cortex in human reasoning: A parametric study of relational complexity. Cerebral Cortex New York NY: 1991, 12(5): 477-485.

Larkin, W. \& Burns, D. 1977. Sentence comprehension and memory for embedded structure. Memory and Cognition 5(1): 17-22.

Lewis, R. 1996. A theory of grammatical but unacceptable embeddings. Journal of Psycholinguistic Research 25: 93-116.

Lewis, R.L., Vasishth, S. \& Van Dyke, J.A. 2006. Computational principles of working memory in sentence comprehension. Trends in Cognitive Sciences 10(10): 447-454.

Lindenberg, R., Fangerau, H. \& Seitz, R.J. 2007. 'Broca's area' as a collective term? Brain and Language 10(21): 22-29.

MacWhinney, B. \& Pleh, C. 1988. The processing of restrictive relative clauses in Hungarian. Cognition 29(2): 95-141.

Mak, W.M., Vonk, W. \& Schriefers, H. 2002. The influence of animacy on relative clause processing. Journal of Memory and Language 47: 50-68.

Mayr, U., Diedrichsen, J., Ivry, R. \& Keele, S.W. 2006. Dissociating task-set selection from taskset inhibition in the prefrontal cortex. Journal of Cognitive Neuroscience 18(1): 14-21.

McNab, F., \& Klingberg, T. 2008. Prefrontal cortex and basal ganglia control access to working memory. Nature Neuroscience 11(1): 103-107.

Miller, G.A. \& Chomsky, N. 1963. Finitary models of language users. In Handbook of Mathemat ical Psychology, Vol. 2, R.D. Lude, R.R. Bush \& E. Galanter (Eds), New York NY: Wiley.

Miyake, A., Carpenter, P.A. \& Just, M.A. 1994. A capacity approach to syntactic comprehension disorders: Making normal adults perform like aphasic patients. Cognitive Neuropsychology 11(6): 671-717.

Miyake, A., Friedman, N.P., Emerson, M.J., Witzki, A.H. \& Howerter, A. 2000. The unity and diversity of executive functions and their contributions to complex 'frontal lobe' tasks: A latent variable analysis. Cognitive Psychology 41(1): 49-100.

\section{2nd proofs}


Monsell, S. 2003. Task switching. Trends in Cognitive Sciences 7(3): 134-140.

Muller, H.M., King, J.W. \& Kutas, M. 1997. Event-related potentials elicited by spoken relative clauses. Cognitive Brain Research 5(3): 193-203.

Novick, J.M., Trueswell, J.C. \& Thompson Schill, S.L. 2005. Cognitive control and parsing. Reexamining the role of Broca's area in sentence comprehension. Cognitive, Affective and Behavioral Neuroscience 5(3): 263-281.

Packard, M.G. \& Knowlton, B.J. 2002. Learning and memory functions of the Basal Ganglia. Annual Review of Neuroscience 25: 563-593.

Paulesu, E., Frith, C.D. \& Frackowiak, R.S. 1993. The neural correlates of the verbal component of working memory. Nature 362(6418): 342-345.

Peelle, J.E., McMillan, C., Moore, P., Grossman, M. \& Wingfield, A. 2004. Dissociable patterns of brain activity during comprehension of rapid and syntactically complex speech: evidence from fMRI. Brain and language 91(3): 315-325.

Poldrack, R.A., Wagner, A.D., Prull, M.W., Desmond, J.E., Glover, G.H. \& Gabrieli, J.D. 1999 Functional specialization for semantic and phonological processing in the left inferior prefrontal cortex. NeuroImage 10(1): 15-35.

Postle, B.R., Brush, L.N. \& Nick, A.M. 2004. Prefrontal cortex and the mediation of proactive interference in working memory. Cognitive, Affective \& Behavioral Neuroscience 4(4): 600-608

Reali, F. \& Christiansen, M.H. 2007. Word chunk frequencies affect the processing of pronomina object-relative clauses. Quarterly Journal of Experimental Psychology 2006 60(2): 161-170.

Roland, D., Dick, F. \& Elman, J.L. 2007. Frequency of basic English grammatical structures: A corpus analysis. Journal of Memory and Language 57(3): 348-379.

Thompson-Schill, S.L., D’Esposito, M., Aguirre, G.K. \& Farah, M.J. 1997. Role of left inferior prefrontal cortex in retrieval of semantic knowledge: A reevaluation. Proceedings of th National Academy of Sciences of the United States of America 94(26): 14792-14797.

Traxler, M.J., Williams, R.S., Blozis, S.A. \& Morris, R.K. 2005. Working memory, animacy, and verb class in the processing of relative clauses. Journal of Memory and Language 53(2): 204-224.

Van Dyke, J.A. \& McElree, B. 2006. Retrieval interference in sentence comprehension. Journal of Memory and Language 55(2): 157-166.

Wager, T.D., Jonides, J. \& Smith, E.E. 2006. Individual differences in multiple types of shifting attention. Memory \& Cognition 34(8): 1730-1743.

Wager, T.D. \& Smith, E.E. 2003. Neuroimaging studies of working memory: A meta-analysis. Cognitive, Affective \& Behavioral Neuroscience 3(4): 255-274.

Watson, D., Breen, M. \& Gibson, E. 2006. The role of syntactic obligatoriness in the production of intonational boundaries. Journal of Experimental Psychology: Learning, Memory, and Cognition 32(5): 1045-1056.

Watson, D. \& Gibson, E. 2004. The relationship between intonational phrasing and syntactic structure in language production. Language and Cognitive Processes, 196, 713-755.

Weckerly, J. \& Kutas, M. 1999. An electrophysiological analysis of animacy effects in the processing of object relative sentences. Psychophysiology 36(5): 559-570.

Yeung, N. Nystrom, L.E., Aronson, J.A. \& Cohen, J.D. 2006. Between-task competition and cognitive control in task switching. The Journal of neuroscience : the official journal of the Society for Neuroscience, 265, 1429-1438.

Zelazo, P.D. 2006. The dimensional change card sort DCCS: A method of assessing executive function in children. Nature Protocols 1(1): 297-301.

Zelazo, P.D. \& Frye, D. 1998. Cognitive complexity and control: II. The development of executive function in childhood. Current Directions in Psychological Science 7(4): 121-126.

\section{2nd proofs}

